lumen due to vessel wall disease, or stenosis, is traditionally quantified by the maximal degree or highest percentage that the lumen is decreased from the expected normal diameter. The current emphasis on this measure of lumen diameter restriction ignores numerous other features of the lesion, including the basic physics of fluid flow. Determining the impact of an arterial lesion on distal flow in a vessel is critical, as ischemia is the key pathophysiologic event and, ultimately, most relevant to expected clinical outcomes. Fractional flow (FF), or the hemodynamic impact of an arterial lesion, is a novel concept and diagnostic parameter in cerebrovascular disorders, adapted from the coronary circulation as an improved approach to assessing the risk of myocardial ischemia. This overview provides an introduction to FF in cerebrovascular disorders, detailing the potential unique applications to stroke and describing our recent initial work on FF inspired by cardiology.

\section{Cerebrovascular Pathophysiology}

Diverse mechanisms are operative in ischemic stroke patients. Downstream ischemia may result from a combination of events, including plaque disruption, thrombosis, distal embolization, and hypoperfusion or flow effects, the latter involving multiple, complimentary sources of flow. The collateral circulation that compensates for the limitation in antegrade, or forward, flow across the arterial lesion is highly influential, varying with lesion location, plaque architecture, and native anatomical configurations [1]. These seemingly distinct mechanisms actually interact with each other in myriad fashion. Neuroimaging advances and innovative postprocessing techniques now facilitate detailed study of flow across an arterial lesion and the quantification of downstream perfusion using a variety of invasive and noninvasive modalities [2]. Tissue perfusion at the microcirculatory level may also be quantified, yet the source of such flow and relative dependence on collaterals varies. Unraveling or deconstructing the source of tissue perfusion, whether from antegrade or collateral sources, may be difficult when a stenosis, rather than complete occlusion, is identified. The relative contribution of each to tissue perfusion may be important as this may determine the risk of subsequent ischemia, ranging from no-risk to high-risk arterial lesions. Few studies to date have systematically assessed this complex pathophysiology and the related clinical risk in patients with intracranial atherosclerotic disease (ICAD), one of the most common causes of stroke worldwide, or acute ischemic stroke (AIS) patients with occlusive lesions. FF provides a logical and comprehensive imaging parameter that incorporates numerous features of an arterial lesion by measuring its effect on downstream flow.

\section{Neuroimaging of Cerebrovascular Pathophysiology}

Each currently available neuroimaging test identifies a different aspect of cerebrovascular pathophysiology. Novel vessel wall imaging approaches such as isotropic high-resolution magnetic resonance imaging (MRI) may visualize atherosclerotic plaque contents, tandem, multifocal or diffuse lesions, degree of luminal stenosis, surface irregularity, and thrombotic propensity [3]. Transcranial Doppler ultrasonography may identify distal emboli in the downstream territory. Antegrade flow may be quantified by FF measures to reflect local changes in blood flow at the site of a vascular lesion, and computed tomography (CT) or MR perfusion imaging or digital subtraction angiography (DSA) may be used to quantify downstream perfusion. Finally, the extent of collateral sources may be defined by the configuration of proximal collateral routes at the circle of Willis or with the use of scales for grading leptomeningeal collaterals on CT angiography (CTA) or DSA [2]. 
Current neuroimaging capabilities can provide a clear snapshot of an individual patient's immediate state of ischemic pathophysiology, or 'ischemic profile', but this requires assessment of the arterial lesion and the state of downstream perfusion. Such a comprehensive approach applies to stable patients with ICAD or unstable patients with an acute occlusive lesion. The goal to be realized is the creation of profiles of ischemic pathophysiology that will allow stroke neurologists to unravel the major puzzles currently encountered in these patients. For example, now that arterial revascularization strategies abound, the greatest such enigma has been recanalization without reperfusion. One would have expected that removing the arterial occlusion should allow pressure to adequately perfuse downstream tissue, yet resistance has been neglected. Such resistance is not necessarily mediated by arteriolar autoregulation, but by the microcirculation and dynamic changes in the venous circulation in the hours that ensue after arterial occlusion [4]. Another major challenge is to identify patients with symptomatic ICAD at highest risk of stroke, given that recent data reveal that traditional approaches to identifying those at highest risk are not only suboptimal, but have not been applied to the broad expanse of patients with less severe anatomic stenoses who nevertheless are truly at risk [5]. Neuroimaging techniques now provide the ability to study the pathophysiology of stroke in concert with routine clinical practice. This opportunity mandates reconsideration of the standard paradigms that were established without the use of imaging.

\section{Limits of Neuroimaging in Its Current Application}

High-resolution isotropic MRI of the vessel wall is not in routine use due to the lack of standard techniques and limited resolution, preventing quantification of high-risk plaque components [3]. Furthermore, it remains unknown whether it is the presence or relative composition of a lipid core and other features that are paramount to clinical risk. Despite the lack of standardized techniques currently available and absence of data, clinicians often use descriptive features for plaques to designate 'vulnerable or unstable' lesions. Similarly, distal emboli are frequently invoked as a cause of ischemia, yet supportive data are extremely limited and transcranial Doppler ultrasonography with embolus detection is rarely implemented to confirm such pathophysiology. In fact, most descriptions of 'emboli' come from the assumption that small lesions on diffusion-weighted MRI must be due to distal embolic occlusions. Finally, collateral perfusion is acknowledged as an important factor, yet collaterals remain understudied despite available techniques and scoring methods. Collaterals can dramatically alter outcome and the risk of subsequent ischemia in both AIS and ICAD [1, 6-9]. Perfusion imaging of collateral hemodynamics is also routinely available. Most commonly, the pathophysiology is not pursued or analyzed in detail because of costs or criticisms that such approaches are exploratory fishing expeditions, despite the abundant imaging routinely acquired and yet incompletely analyzed in the care of stroke patients.

\section{Time Clock versus Pathophysiological Clock}

The concept of time windows or epochs of cerebral ischemia has been radically altered by imaging that can now distinguish pathophysiological states irrespective of time. Cerebrovascular disorders have previously been arbitrarily split between AIS and chronic disorders such as ICAD. This artificial separation of AIS and ICAD is inappropriate. ICAD patients may present with AIS, although the severity, ischemic tissue changes, and flow characteristics can be very different from AIS in cardioembolism cases. Neuroimaging is capable of uniquely profiling these disparate pathophysiological states, but is rarely used in such fashion. In 
regions of the world where ICAD is more prevalent, stroke severity may be milder and the decision-making for acute treatment even more complex.

The current paradigm for treatment of AIS is an 'all or nothing, go or no-go' decision for thrombolysis or thrombectomy, often with time since stroke onset figuring heavily in the decision. It is largely assumed that a complete arterial occlusion is present, without consideration of flow physiology beyond identification of the perfusion defect in downstream tissue. Revascularization is often conducted without understanding of how such sudden restoration of blood flow may affect downstream neurovascular structures. Current evaluation and treatment strategies for AIS ignore the possibility of underlying atherosclerosis, focusing largely on thrombolysis or retrieval of presumed clots. Heterogeneous patterns of infarction and degrees of reperfusion may be noted throughout the arterial territory, including hyperperfusion that may lead to hemorrhagic transformation. Other forms of reperfusion injury and infarct growth despite reperfusion may also occur.

The pathophysiology of revascularization may be better understood and ultimately predicted if the hemodynamic status, including $\mathrm{FF}$ and collateral flow, is known before treatment. FF across the lesion may predict the risk of subsequent ischemia and the risk of reperfusion injury, including hemorrhage. This use of FF may eradicate the arbitrary separation of AIS and ICAD, focusing solely on hemodynamics and the interaction with other pivotal mechanisms.

\section{The Limits of Percent Stenosis}

Stenosis has been based on maximal luminal narrowing, separating lesions into categories of severity. A historical description rather than concrete data can explain why percent stenosis dominates the study of AIS and ICAD. Intracranial measurement of stenosis was extrapolated from extracranial carotid atherosclerosis $[10,11]$. Collaterals and the hemodynamic status of such extracranial disease were largely unexplored in large clinical trials and confusion persists about the ideal management of asymptomatic lesions several decades later. Intracranial measurement of percentage stenosis was applied using an extrapolation of the extracranial methodology without consideration of hemodynamic influences. Stenosis severity was assumed to be one of the strongest predictors of subsequent stroke in ICAD until collateral flow and hemodynamics was proven to be more important in the very same patient cohorts [5-9].

Due to simplicity and historical inertia, current practice continues to rely on percent stenosis using various noninvasive and invasive angiographic techniques that reflect quite different aspects of the lesion. In fact, transcranial Doppler ultrasonography, CTA, MR angiography (MRA), and DSA portray different aspects of a stenosis with varying emphasis on anatomical versus functional hemodynamic features of a lesion. In the SONIA trial (Stroke Outcomes and Neuroimaging of Intracranial Atherosclerosis), direct comparison of such techniques using percent stenosis not surprisingly demonstrated poor concordance amongst these various techniques [11]. Even in the setting of AIS, the functional impact of percent stenosis is undefined. Hemodynamic patterns and perfusion lesions vary extensively from case to case due to collaterals.

Lesions with $40-49 \%$ stenosis have been largely deemed as nonexistent disease, yet this approach likely fails to identify patients at risk of ischemia. Those lesions at 50-69\% stenosis are likely quite important, yet prior inconsistencies in clinical trials provide mixed evidence of their risk and response to treatment. The 70-99\% category has increasingly been termed high risk or severe, serving as the main target of potential treatment strategies [10,12]. At the extreme end of the spectrum, those with $100 \%$ or complete occlusion are the main target in AIS. 
Clinical definitions of stenosis severity thus remain largely constrained to the maximal degree of arterial narrowing while ignoring hemodynamic impact. Without hemodynamic data, for example, management of chronic occlusions is in disarray, as some require intervention and others are entirely stable or low risk due to compensated hemodynamics and collateral perfusion. In other patients with AIS, the presence of complete occlusion is assumed, despite common depiction of tram-tracking around clots and treatment of partial occlusions as documented by DSA in many endovascular therapy trials. In ICAD, paradoxically, nearly half of all recurrent strokes occur in patients with 40-69\% stenosis [13]. More severe lesions (70-99\%) that cause patients to present with symptoms are, in fact, demonstrated failures of collateral perfusion. Even within the 70-99\% range, those above $95 \%$ may behave as complete occlusions and harbor diminished risk due to adequate collateral flow. Despite these observations, successive studies continue to focus on targeting the 'high-risk' subset of 70-99\% stenoses [12], although this is likely a small sliver of the entire population at risk.

This overwhelming reliance on stenosis severity, devoid of carefully measured hemodynamic impact, leaves many unresolved questions. It remains unclear what definition or measure of disease is optimal. Diffuse diseases due to lengthy lesions and multifocal stenoses are unexplored. High-risk plaque in 'mild' stenosis that suddenly worsens due to rupture remains unaddressed. Designating culprit lesions in tandem disease, the role of collateral compensation, and the impact of various interventions remain understudied due to the traditional fixation on stenosis severity.

\section{A Needed Emphasis on Hemodynamics}

Hemodynamic measures will lead to tailored approaches since patients will be found to harbor unique ischemic profiles. Currently, revascularization with endovascular techniques almost always seeks to achieve complete recanalization, yet the downstream hemodynamic effects may result in an extreme spectrum of response to such revascularization, from a tremendous therapeutic benefit to devastating injury due to hemorrhage or reperfusion injury $[14,15]$. Stenting of ICAD and stentriever therapy for AIS both achieve extremely high recanalization rates, yet 'fixing' such baseline arterial steno-occlusive lesions may result in extremely variable clinical outcomes. Noninvasive imaging measures of FF or hemodynamic impact may provide more accurate guidance for the management of these patients. Hemodynamic evaluation in acute ischemia using FF measures may avoid the irrelevant distinction of a complete occlusion from subtotal arterial narrowing due to clot or vessel wall disease. This approach may provide for rational treatment of the partial occlusions frequently encountered in AIS and risk stratification for patients with minor or fluctuating neurological deficits, rather than alternative approaches that arbitrarily use clot length and also ignore hemodynamic impact. Future endovascular strategies may thus be 'partial' in their intended effect, with limited recanalization and residual stenoses when a malignant profile or poor hemodynamic status is identified. Avoiding adverse effects of reperfusion is likely to be usefully guided by such hemodynamic profiling of cases.

\section{The Future of Cerebrovascular Assessment}

The pathophysiology of ischemia can now be characterized and monitored in real time while managing patients with AIS and patients at risk of recurrent ischemia due to underlying disorders such as ICAD. FF measures may address many of the unresolved questions and unaddressed mechanisms that persist due to the outdated and narrow traditional definition 
of an arterial stenosis or occlusive lesion. Hemodynamic approaches will become increasingly important as medications and devices become more efficient at removing arterial lesions and averting thrombotic consequences. Understanding hemodynamics may advance selection criteria based on improved risk calculations and periprocedural management from acute to chronic stages.

\section{Cardiology Guideposts}

Implementation of FF measures in cardiology and the move away from traditional definitions of coronary stenosis may be used as a guide for advancing the field of cerebrovascular disorders. Atherosclerosis of the coronary, renal, and peripheral circulation is now routinely evaluated by hemodynamic measures of FF across a stenotic arterial lesion. The cerebral circulation and ICAD remain the last frontier for implementation of FF measures. Based on similarities in myocardial infarction (MI) and stroke, the coronary circulation provides the best analogy for management using FF. In the heart, FF reserve (FFR) was initially measured by floating an invasive pressure wire downstream to an atherosclerotic plaque in the coronary circulation and measuring the distal:proximal pressure ratio (PR) during hemodynamic challenge of reserve capacity. FFR in a normal coronary artery equals 1.0 [16]. An FFR value of 0.8 or less identifies ischemia-causing coronary stenoses with an accuracy of more than $90 \%$. Routine anatomic measures of percent stenosis are unable to discern hemodynamic impact, as substantial overlap exists in the values of percent stenosis in coronary artery lesions with low FFR and in those with normal FFR. Because percent stenosis measured on angiography is not able to distinguish hemodynamically significant from nonsignificant stenoses, this underscores the need for functional evaluation, rather than anatomical measures, to identify high-risk lesions [17].

Large, randomized trials have established FFR as a useful physiological test for the assessment of lesion-specific coronary ischemia. FF measures allow for enhanced clinical decision-making that improves event-free survival, reduces unnecessary revascularization or percutaneous coronary intervention (PCI), and lowers costs [18]. Compared with other noninvasive tests of myocardial ischemia, FFR was able to detect reversible ischemia with positive and negative predictive values of 100 and 88\%, respectively. In the FAME study (Fractional Flow Reserve versus Angiography for Multivessel Evaluation), those who underwent FFR-guided revascularization (as compared with patients undergoing anatomically guided revascularization) experienced lower rates of adverse events, placement of fewer coronary stents, and lower healthcare costs. Measurement of FFR significantly reduced the rate of the composite endpoint of death, nonfatal MI, and repeat revascularization at 1 year [19]. The FAME II study used FFR to study patients with stable coronary artery disease (CAD) considered for PCI, randomizing those with at least one stenosis with FFR $\leq 0.8$ to PCI plus optimal medical therapy or medical therapy alone. Recruitment was halted prematurely because of a difference in the primary endpoint (composite of death, MI, or urgent PCI): $4.3 \%$ in the PCI cohort versus $12.7 \%$ in the medical therapy arm (hazard ratio, HR, 0.32; 95\% confidence interval, CI, 0.19-0.53; $\mathrm{p}<0.001)$. Among patients with stenoses that were not functionally significant (FFR $>0.8$ ), the best available medical therapy alone resulted in an excellent outcome, regardless of angiographic appearance of stenoses [20].

Routine FFR measurement improves outcome by allowing judicious use of stents and prevention of ischemia. PCI of a stenotic lesion inducing ischemia, indicated by FFR $\leq 0.8$, is beneficial because the risk of stenting is outweighed by a reduction in ischemic events. Conversely, stenting of a stenotic lesion not inducing ischemia increases adverse events that exceed the low risks with medical therapy alone. A recent analysis of the FAME II data showed 
that quality of life is significantly improved by FFR-guided PCI, which was economically attractive compared to medical therapy [21].

More recently, FF measures have been used without vasodilator-induced measurement of reserve capacity and noninvasive FF methods have also been introduced using CTA and cardiovascular MRI (CMR). Computational fluid dynamics (CFD) of noninvasive CTA can determine FFR. CFD may allow for more accurate assessment of FFR compared with invasive methods, since arterial flow may be disturbed by the probe during invasive catheterization [22]. The DeFACTO (Determination of Fractional Flow Reserve by Anatomic Computed Tomographic Angiography) investigators performed a multicenter study comparing noninvasive and invasive FFR. FFR on CTA had an accuracy of $73 \%(95 \% \mathrm{CI}, 67-78)$ with a sensitivity of $90 \%(95 \% \mathrm{CI}$, 84-95) and a specificity of 54\% (95\% CI, 46-83) [23]. CMR is emerging as an alternative noninvasive approach for the assessment of myocardial perfusion, because it offers superior spatial resolution, does not involve ionizing radiation, and has the potential to measure myocardial perfusion in absolute terms [24]. High-resolution CMR myocardial perfusion reserve at 3.0 T can be used to detect flow-limiting CAD defined by FFR, using both visual and quantitative analyses. Dynamic 3D-CMR perfusion has been used to assess the efficacy of coronary stenting procedures. Three-dimensional CMR stress perfusion provided high diagnostic accuracy for the detection of functionally significant CAD. Myocardial ischemic burden measurements were highly reproducible and allowed the assessment of CAD severity [25].

\section{Implementing FF for Cerebrovascular Assessment}

The impact of such FF measures of coronary disease published in top-tier journals has been remarkable, replacing anatomy of luminal stenosis with hemodynamics as the method for identifying lesions likely to cause ischemia and outlining a logical sequence of potential applications to stroke and stroke-prone patients. Noninvasive techniques may be particularly useful in patients at risk for stroke. Noninvasive hemodynamic assessment of an atherosclerotic plaque would avoid the risk of invasive angiography, helping stroke clinicians to identify high-risk cases amongst a broader range of percent stenosis (40-99\%) compared with the current emphasis on patients with only $>70 \%$ stenosis. Embracing the perspective of hemodynamics and the translation of FF measures from the heart to the brain conjures a future of improved outcomes, reduced costs, and avoidance of unnecessary endovascular procedures in patients with the most common cause of stroke worldwide.

Moving forward in stroke patients with advanced imaging does not necessarily require new hardware and methods of image acquisition, but may rely on current capabilities, with or without new postprocessing techniques. If we required new machines and methods of image acquisition, advances would be expensive, slow, and unlikely to occur on a widespread basis, causing severe selection bias. There exist wide treasure troves of imaging data in trials and daily practice. Despite our capable tools and rich data, the field of cerebrovascular medicine has yet to trace a new trajectory or perspective in its approach to patients. The challenge exists for stroke specialists to adjust their perspective and take on the challenge of such a complex and shifting paradigm.

\section{Invasive Methods}

Digital Subtraction Angiography

Our analyses of collateral perfusion in the downstream territory on DSA in both WASID (Warfarin-Aspirin Symptomatic Intracranial Disease Study for Stroke) and SAMMPRIS 

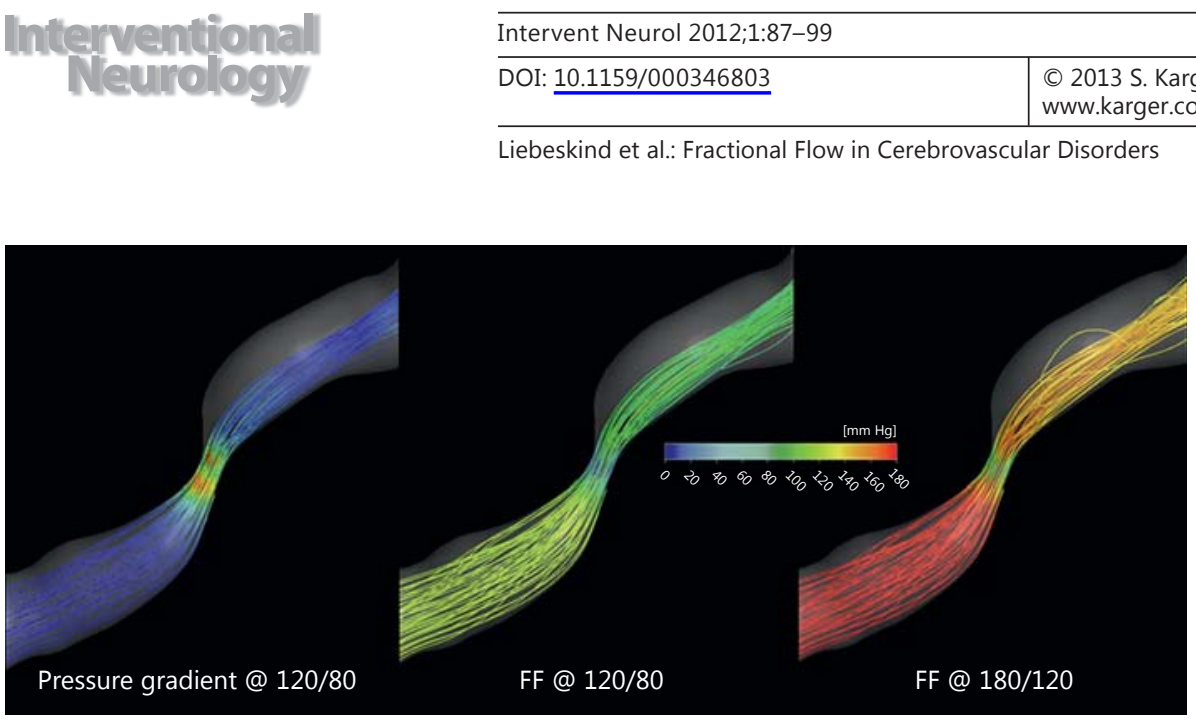

Fig. 1. CFD analyses of an intracranial stenosis from the SAMMPRIS trial, indicating the hemodynamic effects under varying flow conditions.

(Stenting vs. Aggressive Medical Management for Preventing Recurrent Events In Intracranial Stenosis) established the influential role of collaterals as robust risk markers [6-9] yet invasive angiography with adequate injections and prolonged acquisitions is required to identify collaterals. The availability of biplanar acquisitions in SAMMPRIS enabled us to reconstruct 3D geometry from these orthogonal projections and subsequently run CFD analyses of blood flow parameters across the atherosclerotic plaque [26]. Patients with $70-99 \%$ symptomatic stenosis $(n=407)$ had biplanar DSA views available for 3D reconstruction in 249 and CFD simulations in 188 (25 vertebral, 45 basilar, 32 internal carotid, and 86 middle cerebral arteries). Under simulated normal inflow conditions (blood pressure 120/80 mm Hg), only 76/188 (40\%) of these 'severe' stenoses had low FF (fig. 1). Blood pressure fluctuations were also modeled throughout the cardiac cycle to show downstream flow changes.

Despite the ability of CFD to measure pressure gradients in intracranial vessels without placement of a guidewire and pressure transducer, these data were still originally acquired invasively. To maximize the benefit/risk ratio of new, aggressive endovascular or medical interventions for intracranial atherosclerotic disease (IAD), novel noninvasive markers are needed to optimize patient selection. Ideally such markers would be derived from routine imaging studies.

Recent development of a novel postprocessing technique called perfusion angiography now makes it possible to generate perfusion parameter maps directly from conventional angiography (fig. 2) allowing feeding or draining vessels to be simultaneously identified [27]. While not ideally acquired because of the invasive nature of the testing, the data resulting from perfusion angiography could regularly be available in patients being evaluated for endovascular therapy.

\section{Noninvasive Methods}

We developed a novel approach to measure FF that uses routine, noninvasive MRA datasets for hemodynamic prediction of recurrent stroke risk in ICAD. In SONIA, CTA and time-of-flight (TOF) MRA were unreliable in detecting stenosis severity, yet more recent analyses of these same MRA images show that prognostically useful markers can be gleaned without any modification of noninvasive MRA protocols, additional image acquisition, radiation, contrast enhancement, or medication. 

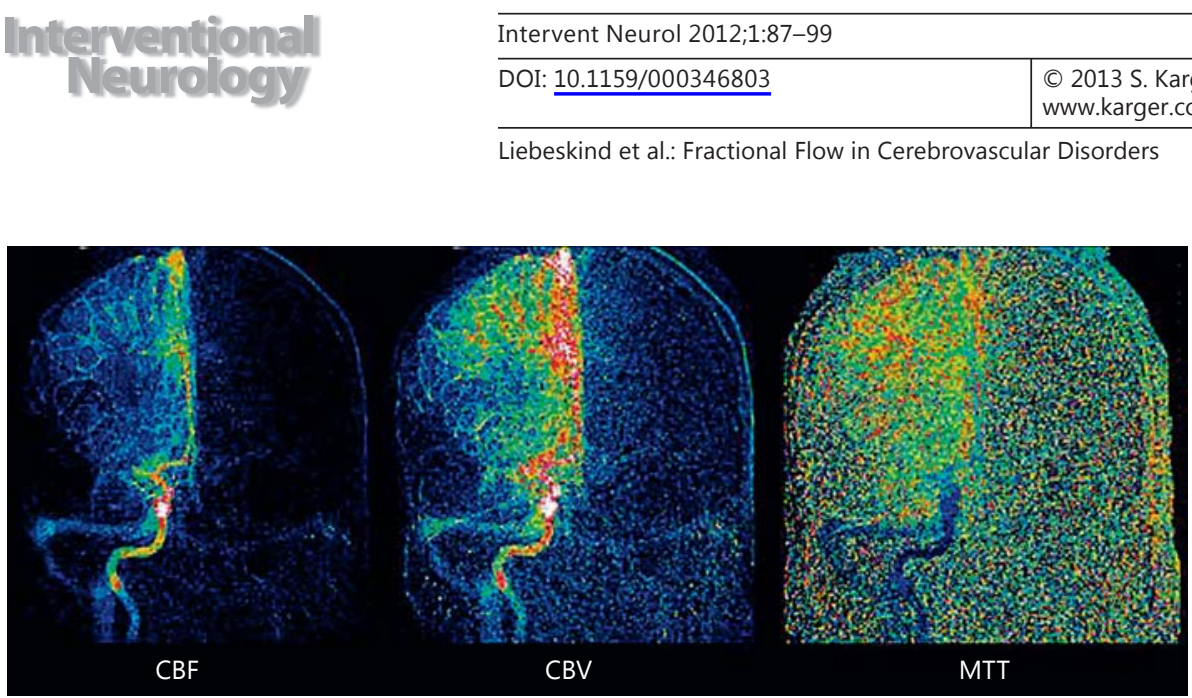

Fig. 2. Perfusion angiography postprocessing of right middle cerebral artery occlusion, revealing downstream hypoperfusion on various parameter maps. $\mathrm{CBF}=$ Cerebral blood flow; $\mathrm{CBV}=$ cerebral blood volume; MTT = mean transit time.

Based on the proportional relationship between blood flow velocity and signal intensity (vessel contrast or flow-related enhancement) on TOF-MRA, we developed a method to systematically measure the hemodynamic effects of an ICAD lesion by measuring the relative distal:proximal signal intensity ratio (SIR) as a marker of FF [28]. The distal:proximal SIR across a stenosis provides an index of hemodynamic significance, where velocity will initially increase in proportion to the degree of cross-sectional area reduction in a vessel, noted as relatively increased signal intensity on MRA. Signal loss and diminished SIR distal to a stenosis occurs with increasingly severe narrowing and hemodynamic impairment of downstream blood flow. This innovative approach actually ignores the degree of stenosis, but incorporates all lesion-specific features that affect flow and avoids the problem of complete loss of the TOF-MRA signal in flow gaps, which occur in nearly $40 \%$ of stenoses [11]. In collaboration with our team in Hong Kong and Beijing, we developed this use of MRA to estimate FF changes across ICAD lesions with various architectures and arterial locations.

SIR was first described as a novel neuroimaging marker of hemodynamic impairment distal to intracranial stenosis by our collaborative group [28]. To determine feasibility and reproducibility, TOF-MRA images of 26 arteries were subjected to SIR analysis. Using a DICOM viewer, a 3-mm region of interest distal and proximal to a stenosis was visualized on maximum intensity projection views. Each region of interest covering the entire vessel lumen was outlined and mean SIR for the region of interest was measured. The mean SIR was $0.84 \pm 0.22$ and intrarater agreement was $88 \%$.

In a further study, 36 patients with moderate/severe stenosis had SIR measurements performed. The patients were followed for 1 year for recurrent stroke in the territory (SIT). The mean SIR was $0.84 \pm 0.23$ and the mean SIR of severe stenoses was not significantly lower than that of moderate stenoses ( 0.80 vs. $0.92, p=0.126$ ). Only 2 patients reached an endpoint of recurrent stroke, limiting the power to test the predictive capability of SIR.

In a third study, tapping an ideal opportunity to validate SIR, we digitized the entire SONIA-WASID MRA imaging archive [5]. Analyses included clinical variables, SIR, and invasive angiography measures (luminal stenosis, thrombolysis in cerebral infarction score of antegrade flow, and collateral grade) to identify predictors of recurrent SIT. Patients with 50-99\% symptomatic ICAD $(n=189)$ had TOF-MRA available. In univariate analysis, HR for SIT of the symptomatic artery with SIR $<0.9$ (SIR below median) was 5.2 (95\% CI, 1.8-15.3; p = 0.001) compared to SIR $\geq 0.9$. In a multivariate analysis correcting for baseline blood pressure, lowdensity lipoprotein, percent stenosis, recency of symptoms, thrombolysis in cerebral infarction, and downstream collaterals, the HR for SIR <0.9 was 10.9 (95\% CI, 2.0-58.9; p = 


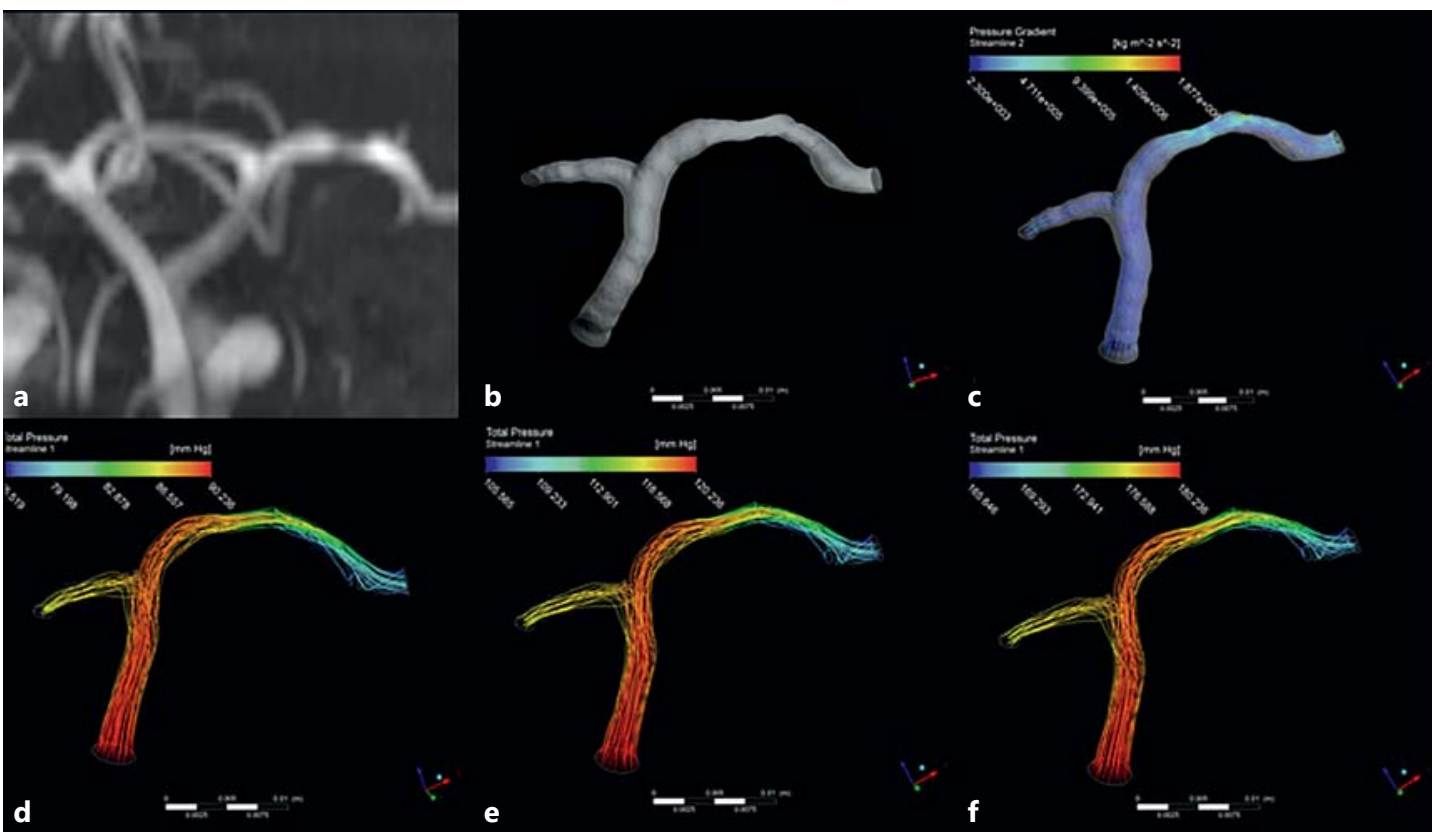

Fig. 3. TOF-MRA CFD analyses of maximum-intensity projection (a), mesh (b), pressure gradient map (c) and PR during hypotensive (d), normal (e), and hypertensive (f) blood flow simulations.

0.001). Only collaterals also had a significant independent association with stroke risk (HR, $13.8,95 \%$ CI, 3.4-55.5; $\mathrm{p}<0.001$ ). In the subset of patients with $<70 \%$ stenosis, an SIR $<0.9$ maintained a significant association with recurrent SIT $(\mathrm{p}=0.006)$, with a 2 -year event rate of $17.3 \%$, showing that even moderate stenoses can pose substantial ischemic risk. We concluded that FF assessed by TOF-MRA SIR may be a useful noninvasive tool to identify highrisk intracranial lesions, even in patients with moderate $(<70 \%)$ degrees of stenosis, and may be suitable for the selection of high-risk patients for clinical trials or aggressive treatments.

Capitalizing on the unique ability of TOF-MRA to depict flow-related geometry, we extended our prior CFD work with SAMMPRIS DSA to noninvasive TOF-MRA. CFD calculations of the PR across various ICAD lesions were simulated under different systemic conditions (fig. 3). CFD simulations were derived from distal:proximal PR at 3 inflow conditions: normal (120/80 $\mathrm{mm} \mathrm{Hg}$ ), hypotensive (90/60 $\mathrm{mm} \mathrm{Hg}$ ), and hypertensive blood flow (180/120 $\mathrm{mm} \mathrm{Hg}$ ). To define the hemodynamic significance of a particular stenosis, abnormal FF was defined as a distal:proximal PR $\leq 0.8$ during the diastolic phase of the cardiac cycle. This stateof-the-art postprocessing technique for FF on TOF-MRA may be performed when flow gaps or apparent discontinuity was noted by visual inspection. Future studies will correlate this hemodynamic index with recurrent SIT.

The FF indices for hemodynamic impairment from TOF-MRA described above, SIR and $\mathrm{PR}$, are complementary noninvasive approaches to predict subsequent stroke. These preliminary studies mirror recent high-impact advances in the cardiac field, adopting FF instead of percent stenosis to identify lesions likely to cause ischemia. These noninvasive TOF-MRA FF measures can be performed with routine image acquisitions, conferring advantages beyond recent cardiac CTA techniques by avoiding the use of radiation or contrast administration. The flow gaps that commonly occur on TOF-MRA previously hampered measures of luminal narrowing in SONIA. The novel perspective embedded in our using of TOF-MRA for FF indices is unhindered by such flow gap issues. 


\section{Serial Imaging}

Multimodal CT/MR are noninvasive imaging tools that provide information regarding antegrade flow across a lesion, antegrade and retrograde collateral flow, tissue perfusion, and aspects of the corresponding venous circulation, all of which may be simultaneously considered as the hemodynamic snapshot of an ischemic profile in an individual patient at a particular point in time [2]. The noninvasive nature of these measures provides a unique opportunity for serial measures over the time course of a patient's illness. Perhaps the simplest example of such an approach would be serial FF measures derived from MRA to follow the evolving ischemic risk of a lesion in a patient with ICAD.

Although a standard CT/MRI protocol is commonly used at most institutions, the specific components or sequences may be tailored to the clinical question. Even further modification of scan parameters may help in answering the specific clinical question. In recent years, postprocessing of noninvasive CTA/MRA and CT perfusion/perfusion-weighted imaging may be performed at the scanner or later to provide detailed information regarding the vascular status. Arterial spin-labeled perfusion imaging uses endogenous labeling of blood flow in the proximal arteries to measure downstream perfusion without the need for exogenous contrast agents [29]. This technique, therefore, holds specific advantage over standard perfusion imaging, as the arterial spin-labeled perfusion imaging can be easily repeated to measure changes in blood flow.

Once images are available for review, interpretation in light of clinical details is critical. The physiological clock of the tissue status available from imaging provides far more information than the previous standard of time from symptom onset. Research in acute stroke imaging has failed, however, to standardize definitions that can be universally applied across all cases, as the evolution of injury may be different in each individual. Serial imaging may depict this aspect of cerebral ischemia in individual patients.

Monitoring the response to various acute stroke therapies may also be facilitated with the use of serial imaging. For instance, resolution of symptoms or rapid improvement may not be due to arterial recanalization, as patients may improve with head-down positioning due to improved residual flow or collateral perfusion despite persisting proximal arterial occlusion. Infarct growth or hemorrhage may be measured with serial or repeated parenchymal imaging. Similarly, recanalization may be chronicled with serial noninvasive angiography techniques, and reperfusion may be illustrated with repeat CT perfusion or perfusionweighted imaging. Recanalization and reperfusion are related, yet distinct components of revascularization may lead to clinical improvement in acute stroke. Serial imaging may provide a window for the better understanding of hemorrhagic transformation and other aspects of reperfusion injury [2].

Single snapshots of the brain and vascular status are generally inferior to serial imaging studies. Such isolated depictions at a single time point, however, also contain footprints of evolving ischemia that may be captured with novel postprocessing techniques. For instance, changes in collateral perfusion may be predicted from the topography embedded within current perfusion imaging techniques, such as those identifying cerebral blood volume gradients [1].

\section{Implications and Future Steps}

Novel imaging approaches in stroke provide great promise and raise new challenges for stroke experts to manage these complex issues. Stroke clinicians must first design clinical trials that actually utilize the pathophysiological data that currently emerge from their 
patients. Previously, trials used guideposts such as time of onset or degree of stenosis that were convenient, but arbitrary, and not reflective of what was actually happening to patients. Perhaps the simplest benefit of an advanced imaging approach is the clarification of unique but realistic pathophysiological states - ischemic profiles - that allow for more consistent patient selection. As an example, a trial assessing ICAD patients only with a particular degree of percent stenosis likely studies a very heterogeneous group of patients in terms of risk for recurrent stroke.

We promote an approach steeped in pathophysiology that may be adapted to study stroke patients who present in diverse ways and to study novel approaches to treatment: patients with diffuse, chronic ICAD, identifying subsets of patients with particularly high recurrent stroke risk and ICAD, studying the effect of varying medical therapies as a function of the patient's ischemic profile, identifying which of the mechanisms currently 'image-able' lead to recurrent symptoms - embolization, flow, or tissue perfusion features. An approach based on pathophysiology would lead to trials that include a broader array of patients than are now being studied, particularly those with 40-69\% ICAD who are at high risk. Trials in AIS could test new algorithms. Should all AIS lesions be revascularized? Should only complete occlusive lesions be revascularized? How much revascularization is enough? When should revascularization be bypassed for an attempt to improve collateral flow or adjust venous resistance to flow? When can the risk for hemorrhagic transformation or reperfusion injury be optimally identified?

Our cousins in cardiology have laid the ground markers. It is up to stroke clinicians and their imaging partners to follow the path, pursue the study of stroke pathophysiology, and set a new trajectory for the evaluation and care of these patients.

\section{Acknowledgments}

D.S.L. was supported by NIH/NINDS P50NS044378 and K24NS072272.

\section{Disclosure Statement}

D.S.L. is a consultant to Stryker, Inc., Covidien, Inc., and CoAxia, Inc. E.F. has no disclosures.

\section{References}

- 1 Liebeskind DS: Understanding blood flow: the other side of an acute arterial occlusion. Int J Stroke 2007;2: 118-120.

2 Liebeskind DS, Alexandrov AV: Advanced multimodal CT/MRI approaches to hyperacute stroke diagnosis, treatment, and monitoring. Ann NY Acad Sci 2012;1268:1-7.

3 Bodle JD, Feldmann E, Swartz RH, et al: High resolution magnetic resonance imaging: an emerging tool for evaluating intracranial arterial disease. Stroke 2013;44:287-292.

4 Liebeskind DS: Collateral perfusion: time for novel paradigms in cerebral ischemia. Int J Stroke 2012;7:309310.

5 Liebeskind DS, Kosinski AS, Lynn MJ, et al: Noninvasive fractional flow on MRA predicts stroke risk of intracranial stenosis in SONIA/WASID. Stroke 2013;44.

6 Liebeskind DS, Cotsonis GA, Saver JL, et al: Collateral circulation in symptomatic intracranial atherosclerosis. J Cereb Blood Flow Metab 2011;31:1293-1301.

7 Liebeskind DS, Cotsonis GA, Saver JL, et al: Collaterals dramatically alter stroke risk in intracranial atherosclerosis. Ann Neurol 2011;69:963-974.

8 Liebeskind DS, Cotsonis GA, Lynn MJ, et al: Collaterals determine risk of early territorial stroke and hemorrhage in the SAMMPRIS trial. Stroke 2012;43:A124. 
9 Liebeskind DS, Cotsonis GA, Lynn MJ, et al: Collateral circulation and hemodynamics of severe intracranial atherosclerosis: angiography and clinical correlates at baseline in the SAMMPRIS trial. Stroke 2012;43:A1900.

10 Chimowitz MI, Lynn MJ, Howlett-Smith H, et al: Comparison of warfarin and aspirin for symptomatic intracranial arterial stenosis. N Engl J Med 2005;352:1305-1316.

11 Feldmann E, Wilterdink JL, Kosinski A, et al: The Stroke Outcomes and Neuroimaging of Intracranial Atherosclerosis (SONIA) trial. Neurology 2007;68:2099-2106.

12 Chimowitz MI, Lynn MJ, Derdeyn CP, et al: Stenting versus aggressive medical therapy for intracranial arterial stenosis. N Engl J Med 2011;365:993-1003.

13 Kasner SE, Chimowitz MI, Lynn MJ, et al: Predictors of ischemic stroke in the territory of a symptomatic intracranial arterial stenosis. Circulation 2006;113:555-563.

14 Bang OY, Saver JL, Kim SJ, et al: Collateral flow averts hemorrhagic transformation after endovascular therapy for acute ischemic stroke. Stroke 2011;42:2235-2239.

15 Bang OY, Saver JL, Kim SJ, et al: Collateral flow predicts response to endovascular therapy for acute ischemic stroke. Stroke 2011;42:693-699.

16 Pijls NH, De Bruyne B, Peels K, et al: Measurement of fractional flow reserve to assess the functional severity of coronary-artery stenoses. N Engl J Med 1996;334:1703-1708.

17 Govindaraju K, Badruddin IA, Viswanathan GN, et al: Evaluation of functional severity of coronary artery disease and fluid dynamics' influence on hemodynamic parameters: a review. Phys Med 2012, E-pub ahead of print.

18 Berger A, Botman KJ, MacCarthy PA, et al: Long-term clinical outcome after fractional flow reserve-guided percutaneous coronary intervention in patients with multivessel disease. J Am Coll Cardiol 2005;46:438-442.

19 Tonino PA, De Bruyne B, Pijls NH, et al: Fractional flow reserve versus angiography for guiding percutaneous coronary intervention. N Engl J Med 2009;360:213-224.

20 De Bruyne B, Pijls NH, Kalesan B, et al: Fractional flow reserve-guided PCI versus medical therapy in stable coronary disease. N Engl J Med 2012;367:991-1001.

21 Saxena A, Min JK, Koo BK, et al: Projected costs and consequences of CT-determined fractional flow reserve (FFRct). J Cardiovasc Comput Tomogr 2012;6:S2.7.

22 Koo BK, Erglis A, Doh JH, et al: Diagnosis of ischemia-causing coronary stenoses by noninvasive fractional flow reserve computed from coronary computed tomographic angiograms. Results from the prospective multicenter DISCOVER-FLOW (Diagnosis of Ischemia-Causing Stenoses Obtained Via Noninvasive Fractional Flow Reserve) study. J Am Coll Cardiol 2011;58:1989-1997.

23 Min JK, Koo BK, Erglis A, et al: Usefulness of noninvasive fractional flow reserve computed from coronary computed tomographic angiograms for intermediate stenoses confirmed by quantitative coronary angiography. Am J Cardiol 2012;110:971-976.

24 Greenwood JP, Maredia N, Younger JF, et al: Cardiovascular magnetic resonance and single-photon emission computed tomography for diagnosis of coronary heart disease (CE-MARC): a prospective trial. Lancet 2012; 379:453-460.

25 Manka R, Paetsch I, Kozerke S, et al: Whole-heart dynamic three-dimensional magnetic resonance perfusion imaging for the detection of coronary artery disease defined by fractional flow reserve: determination of volumetric myocardial ischaemic burden and coronary lesion location. Eur Heart J 2012;33:2016-2024.

26 Liebeskind DS, Fong A, Scalzo F, et al: SAMMPRIS angiography discloses hemodynamic effects of intracranial stenosis: computational fluid dynamics of fractional flow. Stroke 2013;44.

27 Liebeskind DS: Imaging the future of stroke: I. Ischemia. Ann Neurol 2009;66:574-590.

28 Leng XY, Wong KS, Soo YO, et al: Signal intensity ratio as a novel measure of hemodynamic significance for intracranial atherosclerosis. Stroke 2013;44.

29 Chng SM, Petersen ET, Zimine I, et al: Territorial arterial spin labeling in the assessment of collateral circulation: comparison with digital subtraction angiography. Stroke 2008;39:3248-3254. 